\title{
Some new insights into the molecular mechanisms of pain perception
}

\author{
David A. Brown and Gayle M. Passmore
}

Department of Neuroscience, Physiology, and Pharmacology, University College London, United Kingdom.

\begin{abstract}
Bradykinin is the most potent endogenous inducer of acute pain. However, the way in which it excites nociceptive sensory nerve endings is still unclear. In an article recently published in the JCI, Liu et al. suggest a new mechanism via which bradykinin induces acute spontaneous pain. The authors report that the stimulation of $B_{2}$ bradykinin receptors by bradykinin triggers the release of intracellular calcium ions from nociceptive sensory neurons of rat dorsal root ganglia. This depolarizes the sensory nerve endings by simultaneously closing M-type potassium channels and opening TMEM16A chloride channels, resulting in the production of nociceptive signals. Here, we discuss the relationship between this effect and a previously described mechanism for pain sensitization and evaluate its potential significance for therapeutic pain control. A separate study by Patwardhan et al. in this issue of the JCI identifies oxidized linoleic acid metabolites as novel mediators of thermally induced pain.
\end{abstract}

Bradykinin is the most potent endogenous pain-producing substance known $(1,2)$. It is a 9 -amino acid peptide that is clipped off from a $110-\mathrm{kDa}$ precursor plasma $\alpha$-globulin termed kininogen by the serine protease kallikrein (Figure 1). Kallikrein is formed from an inactive precursor, prekallikrein. In damaged tissue, this conversion of prekallikrein to kallikrein is accelerated by clotting factor XII (also known as Hageman factor); this occurs, along with the formation of bradykinin, when the three generator proteins (Hageman factor, prekallikrein, and kininogen) come into contact with negatively charged cell surfaces such as those of endothelial cells (3). Once formed, bradykinin is quite rapidly inactivated in the plasma and lungs by peptidases called kininases, so its effects are largely confined to the tissues where it is formed i.e., it acts as a local inflammatory mediator and induces nocifensive responses.

In the short term at least, the pain-inducing effects of bradykinin are produced by activation of a $G$ protein-coupled receptor termed the $\mathrm{B}_{2}$ receptor, though an additional receptor called the $B_{1}$ receptor may be induced during long-term inflammation. Two components of nocifen-

Conflict of interest: G.M. Passmore has received salary support from Pfizer Global Research and Development.

Citation for this article: J Clin Invest. 2010;

120(5):1380-1383. doi:10.1172/JCI42143. sive action have been identified $(1,2)-$ a direct activation of sensory nerve endings and a sensitization of sensory nerves to other noxious and non-noxious stimuli (termed hyperalgesia and allodynia, respectively). The mechanism of bradykinin-induced hypersensitivity is now quite well understood (4): it is due to increased temperature sensitivity of the transient receptor potential cation channel, subfamily $\mathrm{V}$, member 1 (TRPV1) in sensory axons (see $A$ messenger for the pain induced by noxious heat?). These channels normally respond only to noxious heat (above $42^{\circ} \mathrm{C}$ ), but, after exposure to bradykinin, they respond to much lower, ambient temperatures. (This would explain why, after a sunburn, a normally pleasant shower temperature becomes painful.) However, in spite of much previous work, a universally acceptable mechanism for the direct nociceptive action of bradykinin has not been forthcoming.

\section{The acute response to bradykinin on} sensory nociceptive neurons

In a study recently published in the JCI (5), Liu and colleagues have come up with an attractive explanation for the acute, pain-inducing actions of bradykinin (Figure 2). In sensory neurons (as in other cells), the main effect of stimulating $B_{2}$ bradykinin receptors is to activate the $\mathrm{G}$ protein $\mathrm{Gq}$, the $\alpha$-subunit of which then activates phospholipase $\mathrm{C}$ in the cell membrane to hydrolyze phosphatidylinositol-4,5-bisphosphate ( $\left.\mathrm{PIP}_{2}\right)$. This leads to the release of inositol-1,4,5-trisphosphate $\left(\mathrm{IP}_{3}\right)$ into the cytosol, which in turn releases calcium ions from the endoplasmic reticulum, producing a rise in intracellular $\mathrm{Ca}^{2+}$ concentration. This sequence of events is well known, and Liu et al. show directly - using fluorescent probes for $\mathrm{PIP}_{2}, \mathrm{IP}_{3}$, and $\mathrm{Ca}^{2+}-$ that it occurs in the small nociceptive neurons of rat dorsal root ganglia. The novelty comes in what happens after this. Liu et al. show that the rise in intracellular $\mathrm{Ca}^{2+}$ concentration results in two simultaneous effects on sensory neurons. First, it inhibits a potassium current carried by Kv7.2/Kv7.3 channels, the so-called M-current; this causes the cells to depolarize and to fire more action potentials when challenged with a depolarizing current - i.e., they are more excitable. This itself is not that unexpected (though not previously shown in sensory neurons), since the M-current had already been identified in nociceptive sensory neurons (6), and the authors (7) and others (8) have previously shown that M-current inhibition is the cause of the excitatory action of bradykinin on sympathetic neurons. However, the second effect is new: the authors report that the rise in $\mathrm{Ca}^{2+}$ concentration also activates a calciumdependent chloride current. This adds to the membrane depolarization because, in sensory neurons, as in other peripheral neurons (9), the concentration of intracellular chloride ions is rather high, so that the reversal potential for a chloride current $(-34$ to $-37 \mathrm{mV}$ ) is positive to the resting membrane potential (5). Hence, when chloride channels are opened at the resting potential, chloride ions leave the neuron instead of entering it (as they do in central neurons), producing a net-positive (depolarizing) inward current. As a further point of novelty, Liu et al. (5) identify the bradykinin-induced chloride current as that carried by the recently discovered calcium-activated chloride channel 


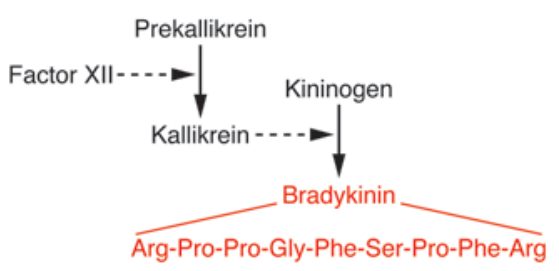

Figure 1

Pathway of bradykinin formation. Bradykinin is formed by the interaction of factor XII, prekallikrein, and high-molecular-weight kininogen. The 9-amino acid sequence of bradykinin is shown in red. See ref. 3 for further details.

TMEM16A (also known as anoctamin 1) (10-12). Thus, TMEM16A was expressed in small TRPV1-postive sensory neurons, as previously noted (10), and a partial siRNA knockdown of Tmem16a reduced the bradykinin-induced current by around $50 \%$. Finally, by blocking the M-channels with the specific blocker XE991 and eliminating the depolarizing $\mathrm{Cl}^{-}$current by altering the $\mathrm{Cl}^{-}$gradient, Liu et al. show that inhibition of M-current and activation of the $\mathrm{Cl}^{-}$current are both sufficient and necessary to explain the increased excitability of these neurons following application of bradykinin.

\section{From neuron somata to peripheral nerve endings}

All of the above conclusions were drawn from the results of experiments on capsaicin-sensitive (hence nociceptive) sensory neurons isolated from young rats and kept in short-term tissue culture. What distinguishes the current work reported by Liu et al. (5) from the numerous preceding (and often contradictory) observations on such neurons is that their conclusions appear to translate quite well to the painful effects of bradykinin on the afferent terminals of these neurons in adult animals. This was assessed by recording the behavioral responses of adult rats to local injections of bradykinin into one hind paw. They found that the effect of bradykinin could be partly replicated, and subsequently attenuated by local injection of the M-channel blocker XE991, and could also be reduced by local application of the specific M-channel opener retigabine. These effects of XE991 and retigabine match other observations of their action on $A \delta$-afferent discharges (the fibers responsible for conveying acute pain) in rat and mouse isolated skin-saphenous nerve preparations $(13,14)$, thus further demonstrating the presence of M-channels in peripheral nociceptive sensory endings. Liu et al. (5) also found that the chloride channel blockers 4,4'-diisothiocyanatostilbene-2,2'-disulphonic acid (DIDS) and 5-nitro-2-(3-phenylpropylamino) benzoic acid (NPPB) strongly reduced the pain-inducing action of intraplantar bradykinin. While not specifically implicating TMEM16A, this implies that chloride channel activation does indeed contribute to the action of bradykinin on peripheral nerve endings and further that these afferent nerve endings have a high intracellular chloride concentration, like the cell bodies. This is supported by a previous observation that GABA (which opens another type of chloride channel) can activate nociceptive afferents in the rat tail (15).

\section{Bifurcating responses to bradykinin mediated by the same receptor}

Thus, we end up with a very interesting dichotomy in the cellular actions of bradykinin, leading to acute pain stimulation on the one hand and thermal pain sensitization on the other (Figure 2). Both events are initiated through activation of $\mathrm{B}_{2}$ receptors and the hydrolysis of $\mathrm{PIP}_{2}$, but they are induced by different second messengers: acute pain is induced by $\mathrm{IP}_{3}$ and the subsequent release of $\mathrm{Ca}^{2+}$, with

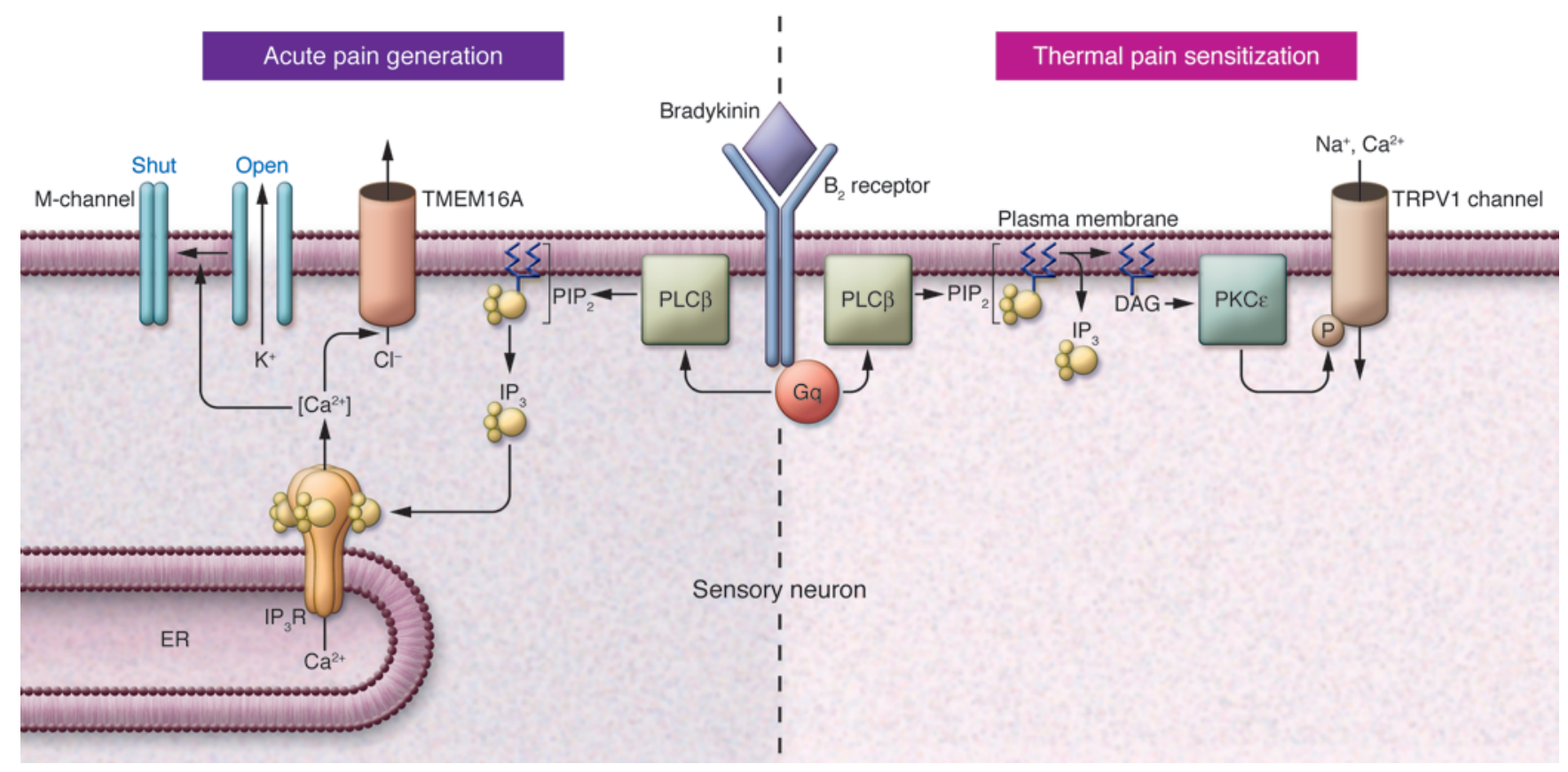

Figure 2

Pain generation and sensitization. The schematic illustrates the bifurcating pathways to acute pain generation (left), as recently reported in the $\mathrm{JCl}$ by Liu et al. (5), and pain sensitization (right; ref. 4), following stimulation of $\mathrm{B}_{2}$ receptors by bradykinin. The two responses result from the generation of different second messengers following $\mathrm{Gq} /$ phospholipase-activated phosphoinositide hydrolysis. $I P_{3} R$, IP 3 receptor; PLC $\beta$, phospholipase $C \beta$. 
consequent inhibition of M-channels and activation of TMEM16A channels; and pain sensitization is induced by diacylglycerol (DAG), activation of the $\mathrm{Ca}^{2+}$-independent protein kinase $\mathrm{PKC} \varepsilon$, and consequent phosphorylation and enhanced thermal sensitivity of TRPV1 channels. This recalls the dual effects of bradykinin via $\mathrm{IP}_{3}$ and DAG previously reported in neuroblastoma cells (16), though the functional end points differ. In principle, TRPV1 sensitization also could contribute to the acute effect of bradykinin if the thermal threshold of TRPV1 channels was reduced to ambient body temperature; and indeed there have been some reports suggesting this (2). However, this is discounted by Liu et al. (5), because TRP channel blockers did not modify either the responses of the sensory neurons or behavioral responses to bradykinin.

\section{Therapeutic implications?}

What about implications for therapy? Although the intracellular chloride concentration may be increased in inflammation (17), and hence the bradykininactivated TMEM16A current augmented, this only forms one component of bradykinin's action, and it only contributes to its acute effect, not its chronic effects. Hence, it is unlikely that drugs that selectively block these channels (if any emerge) will prove to be useful antinociceptives. More promising are the M-channel openers, since they can potentially reduce any form of excitation in nerve cells that possess M-channels, not just excitation that results from $\mathrm{M}$-channel inhibition. Indeed, one such drug (flupirtine) has been in clinical use for some time as an analgesic, and one of the openers used by Liu et al. (5), retigabine, is currently in trial for the treatment of postherpetic neuralgia (18). However, M-channels are widely distributed in both peripheral and central nervous systems, and the M-channel openers so far developed are lipophilic, so they also readily access channels in the central nervous system and hence exert multiple effects. In view of the current evidence for the activity of M-channels in peripheral nerves (19), including that reported by Liu et al. (5), it would be interesting to identify (and test) less lipophilic compounds. Finally, one cannot ignore the other $\left(B_{1}\right)$ bradykinin receptor. This is not involved in the acute action of bradykinin but is induced by nerve and tissue injury and plays a significant role in chronic and neuropathic pain (20). Its modus operandi in such conditions may differ greatly from that of the $\mathrm{B}_{2}$ receptor studied by Liu et al. (5).

\section{A messenger for the pain induced by noxious heat?}

By coincidence, an article in this issue of the journal (21), by Patwardhan et al., addresses the question of how painful heat opens the TRPV1 channels, as depicted in Figure 2. As noted above, the TRPV1 channel is activated at temperatures above $42^{\circ} \mathrm{C}$. Since this effect can be seen in excised membrane patches containing expressed TRPV1 channels (22), it has seemed most likely that temperature exerts a direct effect on the channel protein itself and that the direct thermal activation of these channels in the cutaneous sensory nerve endings was responsible for the response to noxious heat.

However, Patwardhan et al. (21) now suggest that the TRPV1 channels in sensory nerve endings in the skin may not be activated directly by heat, but instead (or at least in part) by what one might term a "heat messenger" released from the surrounding tissue. Following on from previous work on depolarized spinal cord tissue (23), they identify this messenger as linoleic acid (a common membrane fatty acid) that stimulates TRPV1 channels through its oxidation products 9- and 13-hydroxyoctadecadienoic acid (9- and 13-HODE). As evidence that these metabolites contribute to the response of skin nociceptive sensory fibres in situ, the authors show that the latency to foot withdrawal following application of radiant heat to a mouse's paw was increased by locally injecting either a mixture of antibodies to these two metabolites or a compound (nordihydroguaiaretic acid [NDGA]) that inhibits their production from linoleic acid.

More provocatively, the authors also suggest that these linoleic acid metabolites might act as transducers that actually mediate - or at least facilitate - the response of the TRPV1 channels themselves to heat, even in isolated membrane patches (21). Thus, they found that 9-HODE activated TRPV1 channels in excised inside-out membrane patches with a latency comparable to that of an applied heat ramp and, further, that linoleic acid (through conversion to 9/13-HODE) can rescue the response of these TRPV1containing membrane patches to heat after that response had "run down" following patch excision. This is the type of experiment that has previously been crucial in establishing the role of membrane $\mathrm{PIP}_{2}$ as an endogenous regulator of TRPV1 (24) and other (25) ion channels. However, it does not directly show that 9- and 13-HODE actually mediate the primary response to heat (as opposed to facilitating it). For this, we need first to know how accurately they replicate the effects of heat - for example, do they produce changes in the voltage-dependent kinetics of TRPV1 channels comparable with those produced by heat (26). We also need to know whether NDGA or the HODE antibodies inhibit the thermal response of TRPV1 channels in isolated membrane patches (in intact cells, they were only partially effective).

There are several other natural tissue constituents that might act as potential TRPV1-activating messengers (4). To pin down the unique role of linoleic acid, we really need specific HODE antagonists. The studies by Patwardhan et al. $(21,23)$ suggest this to be a worthwhile endeavor, since linoleic acid is released from a variety of cell membranes subject to different forms of trauma. Hence, the development of such compounds may offer new approaches to pain therapy in general.

\section{Acknowledgments}

Work by the authors referred to above was supported by the U.K. Medical Research Council. G.M. Passmore is an Honorary Research Fellow at University College London and Principal Scientist at Pfizer Global Research and Development, Sandwich, Kent, United Kingdom.

Address correspondence to: David A. Brown, Department of Neuroscience, Physiology, and Pharmacology, University College London, Gower Street, London WC1E 6BT, United Kingdom. Phone: 44.0.207.679.7297; Fax: 44.0.207.679.4496; E-mail: d.a.brown@ucl.ac.uk.

1. Dray A, Perkins M. Bradykinin and inflammatory pain. Trends Neurosci. 1993;16(3):99-104.

2. Mizumura K, Sugiura T, Katanozaka K, Banik RK, Kozaki Y. Excitation and sensitization of nociceptors by bradykinin: what do we know? Exp Brain Res. 2009;196(1):53-65.

3. Moreau ME, Garbacki N, Molinari G, Brown NJ, Marceau F. The kallikrein-kinin system: current and future pharmacological targets. J Pharmacol Sci. 2005;99(1):6-38.

4. Huang J, Xiang X, McNaughton PA. Inflammatory pain: the cellular basis of heat hyperalgesia. Curr Neuropharmacol. 2006;4(3):197-206. 
5. Liu B, et al. The acute nociceptive signals induced by bradykinin in rat sensory neurons are mediated by inhibition of $\mathrm{M}$-type $\mathrm{K}^{+}$channels and activation of $\mathrm{Ca}^{2+}$-actived $\mathrm{Cl}^{-}$channels. J Clin Invest. 2010;120(4):1240-1252.

6. Passmore GM, et al. KCNQ/M currents in sensory neurons: significance for pain therapy. J Neurosci. 2003;23(18):7227-7236.

7. Gamper N, Shapiro MS. Calmodulin mediates $\mathrm{Ca}^{2+}$-dependent modulation of $\mathrm{M}$-type $\mathrm{K}^{+}$channels. J Gen Physiol. 2003;122(1):17-31.

8. Jones $\mathrm{S}$, et al. Bradykinin excites rat sympathetic neurons by inhibition of $\mathrm{M}$ current through a mechanism involving $\mathrm{B} 2$ receptors and $\mathrm{G}$ alpha $\mathrm{q} / 11$. Neuron. 1995;14(2):399-405.

9. Adams PR, Brown DA. Actions of gamma-aminobutyric acid on sympathetic ganglion cells. J Physiol. 1975;250(1):85-120.

10. Yang YD, et al. TMEM16A confers receptor-activated calcium-dependent chloride conductance. Nature. 2008;455(7217):1210-1215.

11. Caputo A, et al. TMEM16A, a membrane protein associated with calcium-dependent chloride channel activity. Science. 2008;322(5901):590-594

12. Schroeder BC, Cheng T, Jan YN, Jan LY. Expression cloning of TMEM16A as a calcium-acti- vated chloride channel subunit. Cell. 2008; 134(6):1019-1029.

13. Passmore GM, Brown DA. Effects of M-channel modulators on peripheral excitability in rat hairy skin. Program no. 681.8. 2007 Neuroscience Meeting Planner. San Diego, California, USA: Society for Neuroscience.

14. Roza C, Lopez-Garcia JA. Retigabine, the specific KCNQ channel opener, blocks ectopic discharges in axotomized sensory fibres. Pain. 2008;138(3):537-545.

15. Ault B, Hildebrand LM. GABA, Receptor-mediated excitation of nociceptive afferents in the rat isolated spinal cord-tail preparation. Neuropharmacology. 1994;33(1):109-114.

16. Higashida H, Brown DA. Two polyphosphoinositide metabolites control two $\mathrm{K}^{+}$currents in a neuronal cell. Nature. 1986;323(6086):333-335.

17 Funk K, et al. Modulation of chloride homeostasis by inflammatory mediators in dorsal root ganglion neurons. Mol Pain. 2008;4:32.

18. Wickenden AD, McNaughton-Smith G. Kv7 Channels as targets for the treatment of pain. Curr Pharm Des. 2009;15(15):1773-1789.

19. Lang PM, Fleckenstein J, Passmore GM, Brown DA, Grafe P. Retigabine reduces the excitability of unmyelinated peripheral human axons. Neurophar- macology. 2008;54(8):1271-1278.

20. Ferreira J, et al. Reduced nerve injury-induced neuropathic pain in kinin B1 receptor knock-out mice. JNeurosci. 2005;25(9):2405-2412.

21. Patwardhan AM, et al. Heat generates oxidized linoleic acid metabolites that activate TRPV1 and produce pain in rodents. J Clin Invest. 2010;120(5):1617-1626.

22. Tominaga $\mathrm{M}$, et al. The cloned capsaicin receptor integrates multiple pain-producing stimuli. Neuron. 1998;21(3):531-543.

23. Patwardhan AM, Scotland PE, Akopian AN, Hargreaves KM. Activation of TRPV1 in the spinal cord by oxidized linoleic acid metabolites contributes to inflammatory hyperalgesia. Proc Natl Acad SciU S A. 2009;106(44):18820-18824.

24. Lukacs V, Thyagarajan B, Varnai P, Balla A, Balla $\mathrm{T}$, Rohacs T. Dual regulation of TRPV1 by phosphoinositides. J Neurosci. 2007;27(26):7070-7080.

25. Gamper N, Shapiro MS. Regulation of ion transport proteins by membrane phosphoinositides. Nat Rev Neurosci. 2007;8(12):921-934.

26. Voets T, Droogmans G, Wissenbach U, Janssens A, Flockerzi V, Nilius B. The principle of temperaturedependent gating in cold- and heat-sensitive TRP channels. Nature. 2004;430(7001):748-754.

\title{
Neuronal injury: folate to the rescue?
}

\section{Golo Kronenberg ${ }^{1,2,3}$ and Matthias Endres 1,2}

\begin{abstract}
${ }^{1}$ Klinik und Poliklinik für Neurologie and ${ }^{2}$ Center for Stroke Research Berlin, Charité - Universitätsmedizin Berlin, Berlin, Germany.
${ }^{3}$ Klinik und Hochschulambulanz für Psychiatrie und Psychotherapie, Charité - Universitätsmedizin Berlin, Campus Benjamin Franklin, Berlin, Germany.
\end{abstract}

\begin{abstract}
Strong epidemiological evidence indicates that derangement of single-carbon metabolism has detrimental effects for proper CNS functioning. Conversely, a role for folate supplementation in the treatment and prevention of neurodegenerative and neuropsychiatric disorders remains to be established. In this issue of the JCI, in an elegant series of experiments in rodents, Iskandar and colleagues demonstrate a crucial role of folate in the regeneration of afferent spinal neurons after injury. Probing sequential steps in folate metabolism, from cellular entry to DNA methylation, the authors show that axonal regeneration relies upon the integrity of DNA methylation pathways. These findings provide the first demonstration of an epigenetic mechanism contributing to neurorepair and suggest that manipulation of the methylation milieu may offer promising new therapeutic avenues to promote regeneration.
\end{abstract}

Folic acid, also referred to as folate (the anion form) or vitamin B9, plays a central role in single-carbon metabolism. Specifically, in a reaction dependent on vitamin B12, N5-methyltetrahydrofolate acts as a one-carbon donor in the remethylation of homocysteine to methionine. Dietary intake of methionine as such is insufficient to provide enough methyl groups that are required for cellular methylation reactions. Therefore, the body has

Conflict of interest: The authors have declared that no conflict of interest exists.

Citation for this article: $J$ Clin Invest. 2010; 120(5):1383-1386. doi:10.1172/JCI40764. to rely on the folate-dependent de novo generation of methyl groups via the single-carbon pool. Methionine is further converted to S-adenosylmethionine (SAM), which was discovered in 1953 as the "active methionine" by Catoni (1). The methyl moiety attached to the sulfur atom in SAM is chemically reactive. This explains why SAM is able to serve as the principal methyl donor in most biological transmethylation reactions (e.g., methylation of nucleic acids, proteins, or lipids). The transfer of the methyl group of SAM to an acceptor yields S-adenosylhomocysteine (SAH). Dietary folate deficiency therefore confers a strong decrease in the ratio of SAM to SAH. The ratio of these two compounds regulates the balance of many cellular methylation reactions, not least that of genomic DNA. Accordingly, DNA hypomethylation has been demonstrated experimentally as a consequence of dietary folate deficiency (2).

As a water-soluble vitamin, folate cannot be stored in large amounts in the body. The special importance of folate for CNS functioning is evidenced by the fact that in deficiency states, the brain is relatively spared from folate deficiency as compared with other tissues (3). Uptake of folate across the blood-CSF barrier is primarily accomplished via membranebound folate receptors. Autoantibodies against these receptors may lead to cerebral folate deficiency syndrome, a pediatric neurological disorder characterized by psychomotor retardation, cerebellar ataxia, seizures, and low CSF levels of methyltetrahydrofolate (4). By now, solid evidence has also been assembled that establishes an association between folate metabolism and neural tube defects (5), although underlying mechanisms have not been fully elucidated. Furthermore, low folate status and hyperhomocysteinemia have been implicated as poten- 